University of Wollongong

Research Online

Faculty of Engineering and Information

Faculty of Engineering and Information

Sciences - Papers: Part A

Sciences

$1-1-2012$

\title{
Mechanics of design and model development of CVC-plus roll curve
}

Jianzhong Xu

Northeastern University China

Dianyao Gong

Northeastern University China

Zhengyi Jiang

University of Wollongong, jiang@uow.edu.au

Xianghua Liu

Northeastern University China, liuxh@uow.edu.au

Guodong Wang

Northeastern University China

Follow this and additional works at: https://ro.uow.edu.au/eispapers

Part of the Engineering Commons, and the Science and Technology Studies Commons

Research Online is the open access institutional repository for the University of Wollongong. For further information contact the UOW Library: research-pubs@uow.edu.au 


\title{
Mechanics of design and model development of CVC-plus roll curve
}

\begin{abstract}
The mathematic model of CVC-Plus work roll curve is built. The ratio of the initial shifting value to the target crown is determined, and the mathematical model considering the relationship between the coefficients A2, A3, A4, A5 and is established. According to the theoretical analysis, the distance between the maximum or minimum point of the high order equivalent crown for work roll with CVC-plus roll curve and the rolling central point is the times of the roll barrel length. In general, the initial shifting value of the CVC-plus roll curve is not equal to the initial shifting value of the 3 -order CVC roll curve. The coefficient A1 can also be obtained by optimizing the target function with minimizing the axial force.
\end{abstract}

\section{Keywords}

design, model, mechanics, development, curve, cvc, plus, roll

Disciplines

Engineering | Science and Technology Studies

\section{Publication Details}

Xu, J., Gong, D., Jiang, Z., Liu, X. \& Wang, G. (2012). Mechanics of design and model development of CVCplus roll curve. Advanced Materials Research, 418-420 1158-1166. 


\title{
Mechanics of Design and Model Development of CVC-Plus Roll Curve
}

Xu Jianzhong ${ }^{1}$, Gong Dianyao ${ }^{1}$, Jiang Zhengyi ${ }^{2}$, Liu Xianghua ${ }^{1}$, Wang Guodong ${ }^{1}$

(1. State Key Laboratory of Rolling and Automation, Northeastern University, Shengyang, Liaoning, 110004; 2. School of Mechanical, Materials and Mechatronic Engineering, University of Wollongong, Wollongong NSW 2522, Australia)

\begin{abstract}
The mathematic model of CVC-Plus work roll curve is built. The ratio of the initial shifting value $s_{0}$ to the target crown is determined, and the mathematical model considering the relationship between the coefficients $A_{2}, A_{3}, A_{4}, A_{5}$ and $s_{0}$ is established. According to the theoretical analysis, the distance between the maximum or minimum point of the high order equivalent crown for work roll with CVC-plus roll curve and the rolling central point is the $\sqrt{2}$ times of the roll barrel length. In general, the initial shifting value $s_{0}$ of the CVC-plus roll curve is not equal to the initial shifting value of the 3-order CVC roll curve $s_{0}^{\prime}$. The coefficient $A_{1}$ can also be obtained by optimizing the target function with minimizing the axial force.
\end{abstract}

Keywords CVC mill, quintic roll curve, crown ratio, axial force, mathematical model

Excellent profile and flatness are always targets of the modern rolling technology of hot rolled strip. The roll curve is a key factor of strip crown, the creation of roll curve can make the progress of strip profile and flatness control technology. Using the shifting of the upper and lower work rolls, the target work roll crown can be obtained, which is a key approach to control the strip profile and flatness. CVC, CVC-plus and Smart Crown have the function to control the strip profile and flatness. Previous researchers have conducted the study in the area ${ }^{[1-4]}$, however, there is no systemic or detailed investigation. In this study, the method to design CVC-plus roll curve and the relationship between coefficients of roll curve were built.

\section{CVC-plus unload roll gap function and equivalent crown of work roll}

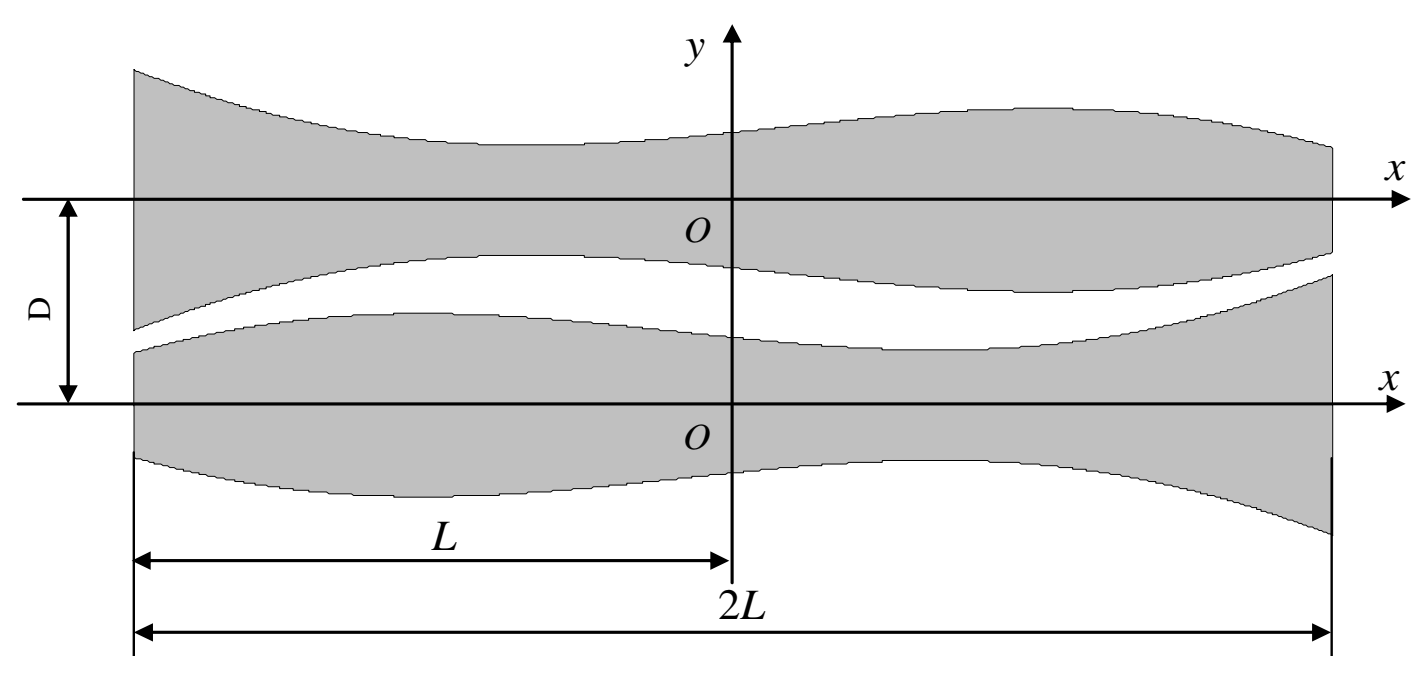

Figure 1 Coordinate system of CVC-plus roll curves

The strip shape defects content not only the central buckle and edge buckle, but also the 1/4 buckle and edge-central composite buckle as well. These shape defects are related to the high order roll gap shape, so the high order roll gap shape tuning should be used to eliminate or improve the shape 
problems. The roll gap of CVC-plus roll consists of both quadratic and high order components. Therefore, the CVC-plus roll can tune the quadratic shape defects, as well as the high order shape defects. The relationship between the roll gap crown and roll shifting value is nonlinear, however, the roll gap crown and high order crown have a linear relationship with the work roll shifting value.

\subsection{Equations of CVC-plus roll curves}

The coordinate system as shown in Figure 1 is selected in this study. $L$ is a half of the roll barrel length (mm), $D$ is the distance between the roll axes. CVC-plus curve is a quintic curve, and the equation of the upper roll curve is listed in Equation (1). As the upper and lower roll curves are anti-symmetric, the CVC-plus roll curve of the lower roll is shown in Equation (2).

$$
\begin{aligned}
& y_{1}(x)=A_{0}+A_{1} x+A_{2} x^{2}+A_{3} x^{3}+A_{4} x^{4}+A_{5} x^{5} \\
& y_{2}(x)=A_{0}-A_{1} x+A_{2} x^{2}-A_{3} x^{3}+A_{4} x^{4}-A_{5} x^{5}
\end{aligned}
$$

\subsection{Models of unloaded roll contour, work roll equivalent crown and crown ratio}

When the upper roll shifts to right for a distance of s, at the same time the lower roll shifts to left for a distance of $s$, the equation of the upper and lower work roll curves, unloaded roll contour and work roll equivalent crown are shown in Equations (3) - (5).

$$
\begin{aligned}
& \left\{\begin{array}{l}
y_{1}(x, s)=A_{0}+A_{1} \times(x-s)+A_{2} \times(x-s)^{2}+A_{3} \times(x-s)^{3}+A_{4} \times(x-s)^{4}+A_{5} \times(x-s)^{5} \\
y_{2}(x, s)=A_{0}-A_{1} \times(x+s)+A_{2} \times(x+s)^{2}-A_{3} \times(x+s)^{3}+A_{4} \times(x+s)^{4}-A_{5} \times(x+s)^{5}
\end{array}\right. \\
& \left\{\begin{aligned}
G(x, s) & =D-y_{1}(x, s)-y_{2}(x, s)=D-2 \times\left(A_{0}-A_{1} \times s+A_{2} \times s^{2}-A_{3} \times s^{3}+A_{4} \times s^{4}-A_{5} \times s^{5}\right) \\
& -2 \times\left(A_{2}-3 \times A_{3} \times s+6 \times A_{4} \times s^{2}-10 \times A_{5} \times s^{3}\right) \times x^{2}-2 \times\left(A_{4}-5 \times A_{5} \times s\right) \times x^{4} \\
\bar{G}(x, s) & =G(x, s)-G(L, s) \\
& =2 \times\left(x^{2}-L^{2}\right) \times\left[\left(10 \times A_{5} \times s^{3}-6 \times A_{4} \times s^{2}+3 \times A_{3} \times s-A_{2}\right)-\left(A_{4}-5 \times A_{5} \times s\right) \times\left(x^{2}+L^{2}\right)\right]
\end{aligned}\right.
\end{aligned}
$$

where $G(x, s)$ is the roll contour, $\bar{G}(x, s)$ is the function of the roll crown distribution.

$$
\left.C_{r}(s)=-\bar{G}(0, s)=2 \times L^{2} \times\left[\left(10 \times A_{5} \times s^{3}-6 \times A_{4} \times s^{2}+3 \times A_{3} \times s-A_{2}\right)-\left(A_{4}-5 \times A_{5} \times s\right) \times L^{2}\right)\right]
$$

As indicated in Equation (5), when $s=0$, the equivalent crown of the work roll is not equal to 0, this means that there is initial shifting value. To assume the initial shifting value is $S_{0}$ when the equivalent crown is 0, and introduces $S_{0}$ into Equation (5):

$$
\left(10 \times A_{5} \times s_{0}^{3}-6 \times A_{4} \times s_{0}^{2}+3 \times A_{3} \times s_{0}-A_{2}\right)-\left(A_{4}-5 \times A_{5} \times s_{0}\right) \times L^{2}=0
$$

There are only two peak values in CVC-plus roll curve which is shown in Equation (1). If the two peak values are $x_{1}$ and $x_{2}$, the following equations can be obtained:

$$
\left\{\begin{array}{l}
y_{1}^{\prime}\left(x_{1}\right)=A_{1}+2 \times A_{2} \times x_{1}+3 \times A_{3} \times x_{1}^{2}+4 \times A_{4} \times x_{1}^{3}+5 \times A_{5} \times x_{1}^{4}=0 \\
y_{1}^{\prime}\left(x_{2}\right)=A_{1}+2 \times A_{2} \times x_{2}+3 \times A_{3} \times x_{2}^{2}+4 \times A_{4} \times x_{2}^{3}+5 \times A_{5} \times x_{2}^{4}=0
\end{array}\right.
$$

Make $y_{1}^{\prime}\left(x_{1}\right)$ minus $y_{1}^{\prime}\left(x_{2}\right)$, considering $\left(x_{2}+x_{1}\right)=-2 \times s_{0}$, the following equation can be obtained: 


$$
A_{2}-3 \times A_{3} \times s_{0}+8 \times A_{4} \times s_{0}^{2}-20 \times A_{5} \times s_{0}^{3}-2 \times\left(A_{4}-5 \times A_{5} \times s_{0}\right) \times x_{1} \times x_{2}=0
$$

Add Equation (6) to Equation (8), Equation (9) is obtained. In general, $\left(x_{2}-x_{1}\right)^{2} \neq 2 \times L^{2}$, therefore, Equation (10) is true. When $A_{4}$ is substituted into Equation (8), the relationship between $A_{2}, A_{3}, A_{5}$ and $s_{0}$ can be shown in Equation (10).

$$
\begin{aligned}
& \left(A_{4}-5 \times A_{5} \times s_{0}\right) \times\left[\left(x_{2}-x_{1}\right)^{2}-2 \times L^{2}\right]=0 \\
& \left\{\begin{array}{l}
A_{4}=5 \times A_{5} \times s_{0} \\
A_{2}=3 \times A_{3} \times s_{0}-20 \times A_{5} \times s_{0}^{3}
\end{array}\right.
\end{aligned}
$$

Substituting $A_{2}$ and $A_{4}$ into Equations (4) and (5), the unloaded roll gap, equivalent crown of work roll and crown ratio of CVC-plus roll curve are shown in Equations (11) (13) respectively. The work roll crown ratio is a ratio of the quadric equivalent crown to the high order equivalent crown. As shown in Equation (12), the distance between the maximum points of the high order equivalent crown and the rolling central point is $\sqrt{2}$ times of the roll barrel length.

$$
\left\{\begin{array}{l}
\bar{G}(x, s)=10 \times A_{5} \times\left(s-s_{0}\right) \times\left(x^{2}-L^{2}\right) \times\left\{x^{2}+\left[L^{2}+2 \times\left(s-s_{0}\right)^{2}+\frac{3 \times A_{3}}{5 \times A_{5}}-6 \times s_{0}^{2}\right]\right\} \\
\bar{G}_{h}(x, s)=10 \times A_{5} \times\left(s-s_{0}\right) \times\left(x^{4}-L^{2} \times x^{2}\right)
\end{array}\right.
$$

where $\bar{G}(x, s)$ is the function of roll gap distribution, $\bar{G}_{h}(x, s)$ is the function of the high order crown distribution.

$$
\begin{aligned}
& \left\{\begin{array}{l}
C_{r}(s)=-\bar{G}(0, s)=10 \times A_{5} \times\left(s-s_{0}\right) \times L^{2} \times\left\{L^{2}+2 \times\left(s-s_{0}\right)^{2}+\left[\frac{3 \times A_{3}}{5 \times A_{5}}-6 \times s_{0}^{2}\right]\right\} \\
\frac{\partial \bar{G}_{h}(x, s)}{x}=10 \times A_{5} \times\left(s-s_{0}\right) \times\left[4 \times x^{3}-2 \times L^{2} \times x\right]=0 \Rightarrow x= \pm \frac{L}{\sqrt{2}} \\
C_{h}(s)=-\bar{G}_{h}(L / \sqrt{2}, s)=\frac{10 \times A_{5} \times\left(s-s_{0}\right) \times L^{4}}{4}
\end{array}\right. \\
& R_{C}(s)=\frac{C_{r}(s)}{C_{h}(s)}=\frac{4}{L^{2}} \times\left\{L^{2}+2 \times\left(s-s_{0}\right)^{2}+\left[\frac{3 \times A_{3}}{5 \times A_{5}}-6 \times s_{0}^{2}\right]\right\}
\end{aligned}
$$

where $C_{r}(s)$ is the equivalent crown of the work roll. $C_{h}(s)$ is the high order crown of the work roll. $R_{C}(s)$ is the crown ratio of the work roll.

\section{Models of computational coefficients of CVC-plus roll curves}

\subsection{Calculation of coefficients $A_{5}-A_{2}$}

(1) Relationship between $A_{2}, A_{3}, A_{4}, A_{5}$ and initial shifting value

If the minimum and maximum shifting values $\left[s_{1}, s_{2}\right]$ and the related equivalent crowns $\left[c_{1}, c_{2}\right]$ are known, 


$$
\left\{\begin{array}{l}
c_{1}=10 \times A_{5} \times\left(s_{1}-s_{0}\right) \times L^{2} \times\left[2 \times\left(s_{1}-s_{0}\right)^{2}+L^{2}+\frac{3 \times A_{3}}{5 \times A_{5}}-6 \times s_{0}^{2}\right] \\
c_{2}=10 \times A_{5} \times\left(s_{2}-s_{0}\right) \times L^{2} \times\left[2 \times\left(s_{2}-s_{0}\right)^{2}+L^{2}+\frac{3 \times A_{3}}{5 \times A_{5}}-6 \times s_{0}^{2}\right]
\end{array}\right.
$$

Coefficient $A_{5}$ can be obtained (see Equation (15)). Considering both Equations (10) and (15), the relationship between $A_{2}, A_{3}, A_{4}, A_{5}$ and initial shifting value $s_{0}$ can be obtained, as shown in Equation (16).

$$
\begin{aligned}
& A_{5}=\frac{\left(c_{2}-c_{1}\right) / s_{2}+\left(c_{2}+c_{1}\right) / s_{0}}{80 \times L^{2} \times\left(s_{0}^{2}-s_{2}^{2}\right)}=\frac{\left(c_{2}-c_{1}\right) \times\left(s_{0}-s_{0}^{\prime}\right)}{80 \times L^{2} \times\left(s_{0}^{2}-s_{2}^{2}\right) \times s_{0} \times s_{2}} \\
& \left\{\begin{array}{l}
A_{4}=5 \times A_{5} \times s_{0} \\
A_{3}=\frac{c_{2}-c_{1}}{6 \times L^{2} \times\left(s_{2}-s_{1}\right)}-\frac{5 \times A_{5} \times\left[L^{2}+2 \times s_{2}^{2}\right]}{3} \\
A_{2}=3 \times A_{3} \times s_{0}-20 \times A_{5} \times s_{0}^{3}
\end{array}\right. \\
& s_{0}=s_{0}^{\prime}=\frac{c_{1}+c_{2}}{c_{1}-c_{2}} \times s_{2} \Leftrightarrow \frac{c_{1}+c_{2}}{s_{0}}=-\frac{c_{2}-c_{1}}{s_{2}}
\end{aligned}
$$

As shown in Equation (15), if the initial shifting value of CVC-plus roll curve is equal to the initial value of CVC cubic roll curve, which is shown in Equation (17), the calculation result indicates that the coefficients $A_{5}$ and $A_{4}$ are 0 , and the CVC-plus roll curve becomes a cubic CVC curve. It is a special instance of CVC curve for CVC-plus curve when $s_{0}=s_{0}^{\prime}$, as well as for the quintic CVC-plus roll curve when $s_{0} \neq s_{0}^{\prime}$. Therefore, how to determine the initial position $s_{0}$ and the coefficient $A_{5}$ become the key problems in the design and control.

(2) Determination of initial shifting value $s_{0}$ and coefficient $A_{5}$

Though $s_{0} \neq s_{0}^{\prime}$, the difference between them is not large. The initial shifting value of the CVC-plus roll curve can be determined according to the target value of the crown ratio $\bar{R}_{C}$ which can be supplied in plant. From Equation (13), we can know that the relationship between the crown ratio and work roll shifting position are quadratic, and when $s=s_{0}$ the $\bar{R}_{C}$ reaches its maximum value. However, the interval of the shifting values $\left[s_{1}, s_{2}\right]$ is very small. Therefore, when the shifting value is the maximum value $s_{1}$ and $s_{2}$, the target crown ratio $\bar{R}_{C}$ is shown in Equation (18).

$$
\bar{R}_{C}=\frac{\left[R_{C}\left(s_{2}\right)+R_{C}\left(s_{1}\right)\right]}{2}=\frac{4}{L^{2}} \times\left\{L^{2}+\left(s_{2}-s_{0}\right)^{2}+\left(s_{1}-s_{0}\right)^{2}+\frac{3 \times A_{3}}{5 \times A_{5}}-6 \times s_{0}^{2}\right\}
$$

When $c_{1} \times c_{2} \neq 0$, from Equations (15) and (16), the following equation can be obtained,

$$
\frac{3 \times A_{3}}{5 \times A_{5}}=\frac{4 \times\left(s_{0}^{2}-s_{2}^{2}\right) \times s_{0}}{s_{0}-s_{0}^{\prime}}-L^{2}-2 \times s_{2}^{2}
$$

Substitute Equation (19) into Equation (18), the following equation can be obtained: 


$$
\bar{R}_{C}=\frac{16 \times s_{0} \times\left(s_{0} \times s_{0}^{\prime}-s_{2}^{2}\right)}{L^{2} \times\left(s_{0}-s_{0}^{\prime}\right)} \Leftrightarrow 16 \times s_{0}^{\prime} \times s_{0}^{2}-\left[L^{2} \times \bar{R}_{C}+16 \times s_{2}^{2}\right] \times s_{0}+s_{0}^{\prime} \times L^{2} \times \bar{R}_{C}=0
$$

To solve the equation, Equation (21) must be true. When $\left|s_{0}^{\prime}\right| \leq\left|s_{2}\right|, \quad \Delta_{1} \leq 0 \Rightarrow \Delta \geq 0$, so the crown ratio is random. When $\left|s_{0}^{\prime}\right|>\left|s_{2}\right|, \Delta_{1}>0$.It can be proved that

$$
16 \times\left(2 \times s_{0}^{\prime 2}-s_{2}^{2}-2 \times\left|s_{0}^{\prime}\right| \times \sqrt{s_{0}^{\prime 2}-s_{2}^{2}}\right) / L^{2}>0,
$$

and the maximum value is $16 \times s_{2}^{2} / L^{2} \leq 1$. Therefore, the available interval can be seen in Equation (22). The calculation formula of the initial work roll shifting value and coefficient $A_{5}$ is shown in Equation (23).

$$
\begin{aligned}
& \left\{\begin{array}{l}
\Delta=L^{4} \times \bar{R}_{C}^{2}-32 \times L^{2} \times\left[2 \times s_{0}^{\prime 2}-s_{2}^{2}\right] \times \bar{R}_{C}+16^{2} \times s_{2}^{4} \geq 0 \\
\Delta_{1}=64^{2} \times L^{4} \times s_{0}^{\prime 2} \times\left(s_{0}^{\prime 2}-s_{2}^{2}\right) \\
\bar{R}_{C}=\frac{16}{L^{2}} \times\left[2 \times s_{0}^{\prime 2}-s_{2}^{2} \pm 2 \times\left|s_{0}^{\prime}\right| \times \sqrt{s_{0}^{\prime 2}-s_{2}^{2}}\right] \quad \Leftarrow\left|s_{0}^{\prime}\right|>\left|s_{2}\right|
\end{array}\right. \\
& \left\{\begin{array}{l}
\bar{R}_{C} \neq 0 \\
\bar{R}_{C}>\frac{16}{L^{2}} \times\left[2 \times s_{0}^{\prime 2}-s_{2}^{2}+2 \times\left|s_{0}^{\prime}\right| \times \sqrt{s_{0}^{\prime 2}-s_{2}^{2}}\right] \\
\bar{R}_{C}<0
\end{array}\right\} \Leftarrow\left|s_{0}^{\prime}\right|>\left|s_{2}\right| \\
& \left\{\begin{array}{l}
s_{0}=\frac{\left[L^{2} \times \bar{R}_{C}+16 \times s_{2}^{2}\right] \mathrm{m} \sqrt{\left[L^{2} \times \bar{R}_{C}+16 \times s_{2}^{2}\right]^{2}-64 \times L^{2} \times s_{0}^{\prime 2} \times \bar{R}_{C}}}{32 \times s_{0}^{\prime}} \\
\bar{R}_{C}>0 \text { is" }-" ; \bar{R}_{C}<0 \text { is" }+" \\
A_{5}=\frac{\left(c_{2}-c_{1}\right) / s_{2}+\left(c_{2}+c_{1}\right) / s_{0}}{80 \times L^{2} \times\left(s_{0}^{2}-s_{2}^{2}\right)}
\end{array}\right.
\end{aligned}
$$

When $c_{1} \times c_{2}=0$ or $c_{1}+c_{2}=0, s_{0}=s_{0}^{\prime}$, the coefficient $A_{5}$ can be calculated as Equation (24) due to Equations (13) and (14).

$$
\left\{\begin{array}{l}
s_{0}=s_{0}^{\prime}=\frac{c_{1}+c_{2}}{c_{1}-c_{2}} \times s_{2} \\
A_{5}=\frac{C_{2}-c_{1}}{5 \times L^{4} \times s_{2} \times R_{C}}
\end{array}\right.
$$

\subsection{Determination of roll curve coefficient $A_{1}$ and the roll diameter peak value}

(1) The interval of coefficient $\boldsymbol{A}_{\mathbf{1}}$

Assume the two peak values of Equation (1) are $x_{1}$ and $x_{2}$, then, 


$$
\begin{aligned}
& \begin{aligned}
y_{1}^{\prime}(x)= & A_{1}+2 \times A_{2} \times x+3 \times A_{3} \times x^{2}+4 \times A_{4} \times x^{3}+5 \times A_{5} \times x^{4} \\
= & \left(x-x_{1}\right) \times\left(x-x_{2}\right) \times\left(a \times x^{2}+b \times x+c\right) \\
= & a \times x^{4}+\left[b+2 \times a \times s_{0}\right] \times x^{3}+\left[a \times x_{1} \times x_{2}+2 \times b \times s_{0}+c\right] \times x^{2} \\
& +\left[b \times x_{1} \times x_{2}+2 \times s_{0} \times c\right] \times x+c \times x_{1} \times x_{2}=0
\end{aligned} \\
& \begin{cases}a= & 5 \times A_{5} \\
b= & 4 \times A_{4}-2 \times 5 \times A_{5} \times s_{0}=10 \times A_{5} \times s_{0} \\
c= & 3 \times A_{3}-20 \times A_{5} \times s_{0}^{2}-5 \times A_{5} \times x_{1} \times x_{2} \\
A_{2}= & 3 \times A_{3} \times s_{0}-20 \times A_{5} \times s_{0}^{3} \\
A_{1}= & {\left[3 \times A_{3}-20 \times A_{5} \times s_{0}^{2}-5 \times A_{5} \times x_{1} \times x_{2}\right] \times x_{1} \times x_{2}}\end{cases}
\end{aligned}
$$

The interval of $A_{1}$ is:

$$
\left\{\begin{array}{l}
\frac{d A_{1}}{d\left(x_{1} \times X_{2}\right)}=0 \Rightarrow x_{1} \times x_{2}=\frac{3 \times A_{3}-20 \times A_{5} \times s_{0}^{2}}{10 \times A_{5}}=\frac{1}{2} \times\left[\frac{3 \times A_{3}}{5 \times A_{5}}-4 \times s_{0}^{2}\right] \\
A_{1 \text { PEAK }}=\frac{5 \times A_{5}}{4} \times\left[\frac{3 \times A_{3}}{5 \times A_{5}}-4 \times s_{0}^{2}\right]^{2} \\
-\frac{5 \times\left|A_{5}\right|}{4} \times\left[\frac{3 \times A_{3}}{5 \times A_{5}}-4 \times s_{0}^{2}\right]^{2} \leq A_{1} \leq \frac{5 \times\left|A_{5}\right|}{4} \times\left[\frac{3 \times A_{3}}{5 \times A_{5}}-4 \times s_{0}^{2}\right]^{2} \\
{\left[3 \times A_{3}-20 \times A_{5} \times s_{0}^{2}\right]^{2}-20 \times A_{5} \times A_{1}>0}
\end{array}\right.
$$

(2) Determination of coefficient $A_{1}$

The coefficient $A_{1}$ has a close relationship with the roll diameter peak value, and it determines the axial force of the strip acting on the roll. Considering the strip width, the roll shifting value and the off tracking of strip, using the minimized axial force as the target function, the coefficient $A_{1}$ is optimized $^{[5-10]}$.

Assume the off tracking of strip is $t \in\left[t_{1}, t_{2}\right] . t_{1}$ and $t_{2}$ are the peak values of the off tracking of strip. The axial force on the strip with a width of $2 b$ can be obtained by integral calculation.

$$
\begin{aligned}
& F_{2}=\int_{y_{1}(-b+t, s)}^{y_{1}(b+t, s)} p_{0} d y= p_{0} \times\left[y_{1}(b+t, s)-y_{1}(-b+t, s)\right] \\
&=2 \times b \times p_{0} \times\left\{\begin{array}{c}
A_{1}+2 \times A_{2} \times(t-s)+A_{3} \times\left[3 \times(t-s)^{2}+b^{2}\right] \\
+4 \times A_{4} \times\left[(t-s)^{3}+(t-s) \times b^{2}\right] \\
+A_{5} \times\left[5 \times(t-s)^{4}+5 \times(t-s)^{2} \times b^{2}+b^{4}\right]
\end{array}\right\}
\end{aligned}
$$

where $b$ is a half of the strip width, and $y_{1}(x, s)$ is the roll curve of the upper roll.

(3) Determination of peak value of roll diameter

When the coefficients are determined, the coordinates of the maximum value can be calculated as follows: 


$$
\left\{\begin{array}{l}
x_{1} \times x_{2}=0.5 \times\left[\frac{3 \times A_{3}}{5 \times A_{5}}-4 \times s_{0}^{2}\right] \mathrm{m} \sqrt{\left[\frac{3 \times A_{3}}{5 \times A_{5}}-4 \times s_{0}^{2}\right]^{2}-\frac{4 \times A_{1}}{5 \times A_{5}}} \\
\bar{R}_{C}>0 \text { is" }-" ; \bar{R}_{C}<0 \text { is" }+" \\
x_{1}=-s_{0}-\sqrt{s_{0}^{2}-x_{1} \times x_{2}} \\
x_{12}=-s_{0}+\sqrt{s_{0}^{2}-x_{1} \times x_{2}}
\end{array}\right.
$$

\section{Design of CVC-plus roll curve}

According to the condition of a certain steel plant, using the CVC-plus roll curve mathematical model, the quintic CVC roll curve is determined. The parameters needed for the calculation are listed in Table 1. The calculated coefficients are listed in Table 2. The work roll curve is shown in Figure 2. The relationship between the equivalent crown and the shifting value of the work roll is shown in Figure 3. The profile of the roll gap is shown in Figure 4.

Table 1 Parameters needed for calculation

\begin{tabular}{|c|c|c|}
\hline Parameters & Value & Unit \\
\hline Work roll barrel length & 1650 & $\mathrm{~mm}$ \\
\hline Work roll named diameter & 710 & $\mu \mathrm{m}$ \\
\hline Max. equivalent crown & 165 & $\mu \mathrm{m}$ \\
\hline Min. equivalent crown & -245 & $\mathrm{~mm}$ \\
\hline Shifting area & \pm 100 & $\mathrm{~mm}$ \\
\hline Strip width & $850-1300$ & - \\
\hline Crown ratio & -8.5 & \\
\hline
\end{tabular}

Table 2 Parameters of roll curve

\begin{tabular}{|c|c|}
\hline CVC roll curve parameter & Calculative result \\
\hline$A_{0}$ & 355.00 \\
\hline$A_{1}$ & $-2.264606120865497 \mathrm{E}-004$ \\
\hline$A_{2}$ & $4.248272242897413 \mathrm{E}-008$ \\
\hline$A_{3}$ & $7.088796266017358 \mathrm{E}-010$ \\
\hline$A_{4}$ & $-1.768501519093894 \mathrm{E}-014$ \\
\hline$A_{5}$ & $-1.771761012180488 \mathrm{E}-016$ \\
\hline Initial shifting value $S_{0}$ & 19.963206176632340 \\
\hline Coefficient of axial force $Z_{\mathrm{E}}$ & $8.912790141505940 \mathrm{E}-002$ \\
\hline Radius of left end $R_{\mathrm{WL}}$ & 354.877218672079600 \\
\hline Radius of right end $R_{\mathrm{WR}}$ & 355.164225753436000 \\
\hline Coordinate of minimum point $x_{\min }$ & -354.627623386854300 \\
\hline Radius of maximum point $R_{\mathrm{Wmax}}$ & 354.954313243425400 \\
\hline Coordinate of extreme point $x_{\max }$ & 314.701211033589600 \\
\hline Radius of minimum point $R_{\mathrm{Wmin}}$ & 355.054751093513900 \\
\hline
\end{tabular}




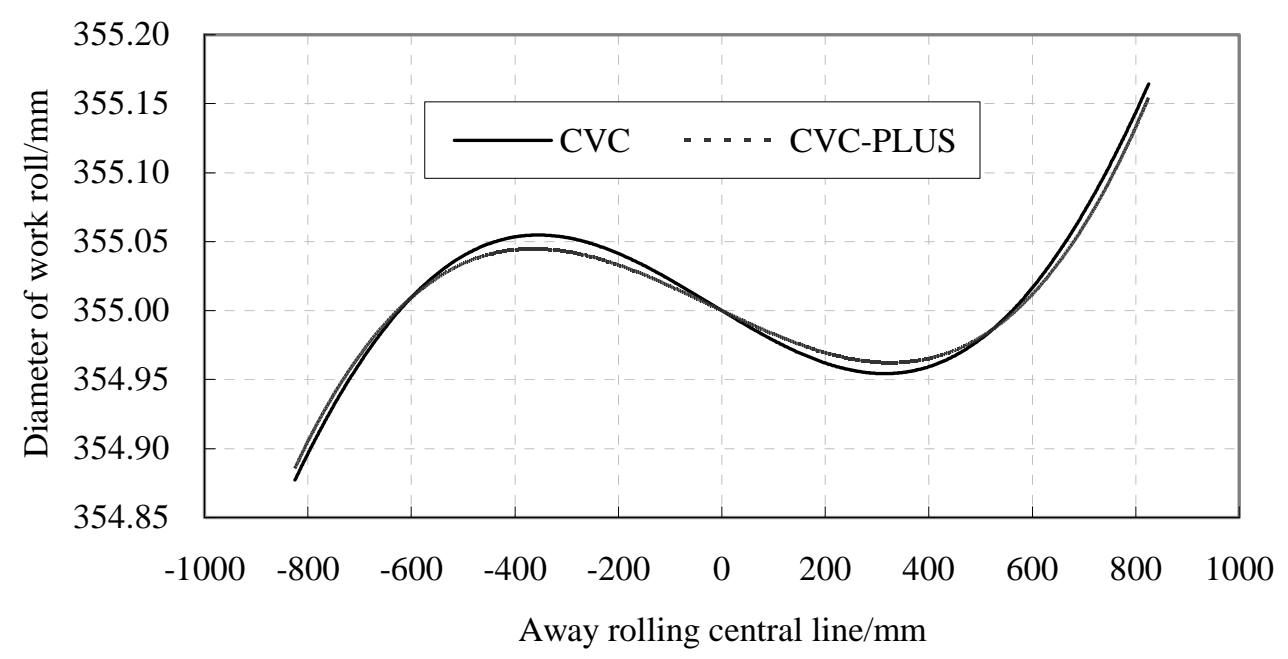

Figure 2 Work roll curves

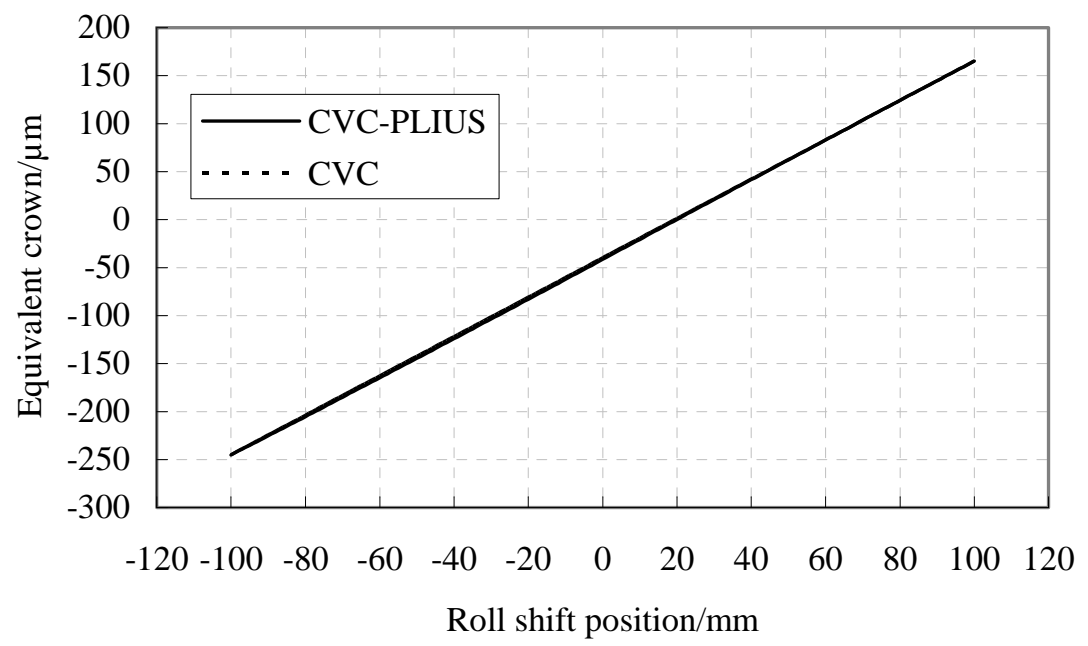

Figure 3 Relationship between equivalent crown and shifting value

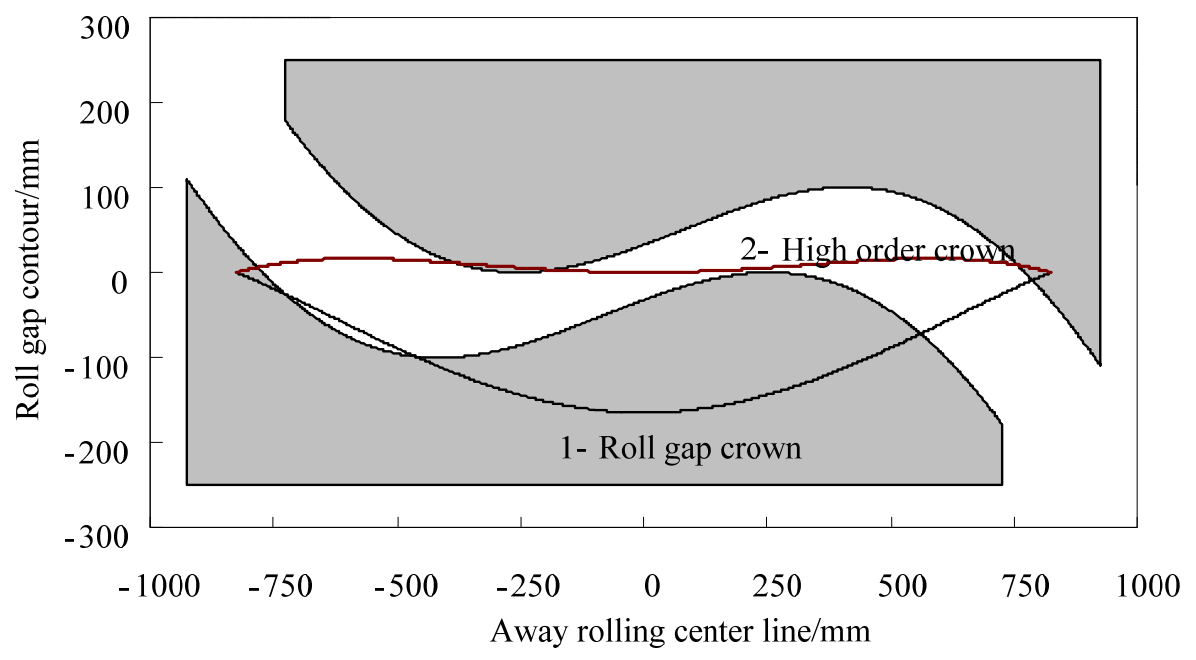

(a) Shifting value is $100 \mathrm{~mm}$ 


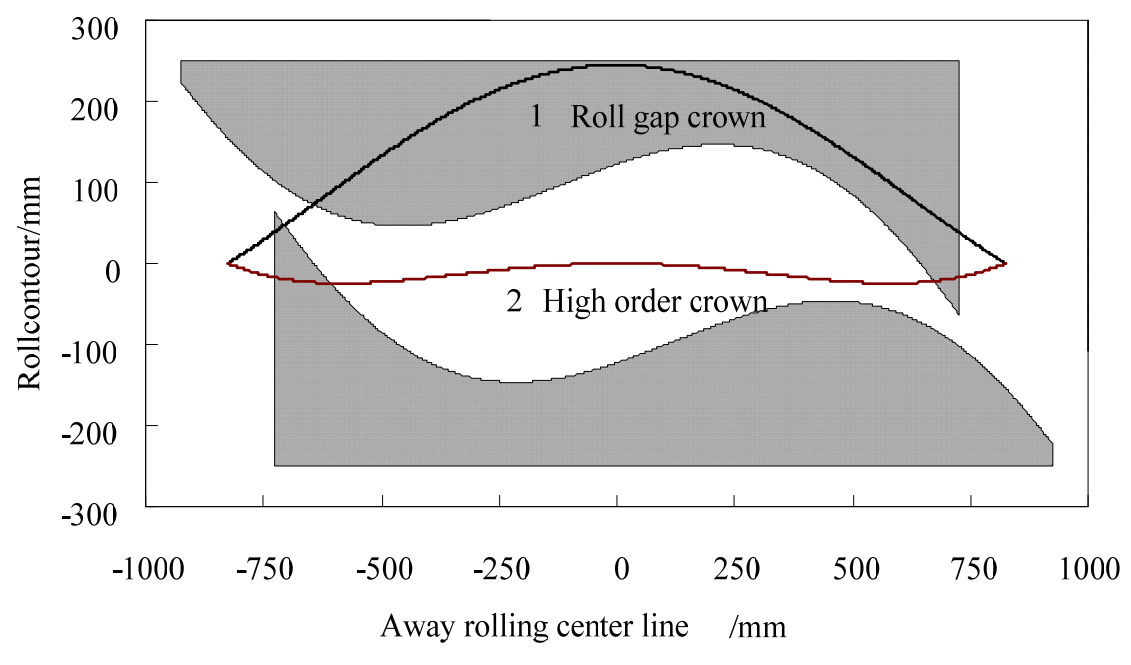

(b) Shifting value is $-100 \mathrm{~mm}$

Figure 4 Roll profile of CVC-plus roll

\section{Conclusions}

1) The distance between the maximum or minimum points of the high order equivalent crown and rolling central point is the $\sqrt{2}$ times of the roll barrel length.

2) In general, the initial shifting value of CVC-plus roll curve is not equal to the initial shifting value of the 3 order CVC roll curve.

3) The relationship between the initial shifting value and the target crown ratio was determined.

4) The relationship between the coefficients $A_{2}, A_{3}, A_{4}, A_{5}$ and $s_{0}$ was built up.

5) The coefficient $A_{1}$ can be obtained by optimization design with the minimized target function of the axial force.

\section{References}

[1] Bald W, Beisemann G, Feldmann H, et al(1987) Continuously Variable Crown (CVC) Rolling. Iron and Steel Engineer. 64 (3): 32

[2] Bald W, Klamma K(1988) CVC Technology for Cold Rolling Mills Plant Example. Iron Steel Engineer. 65 (5): 24

[3] Zhang Jie, Chen Xianlin. Roll(1989) China patent: 89202773.8 ,9

[4] Jiang Z L, Wang G D, Zhang Q, et al(1993) Shifting Roll Profile and Control Characteristics. Journal of Materials Processing Technology. 37: 53.

[5] Lu C, Kiet Tieu A, Jiang ZY, et al(2002) A Design of a Third-order CVC Roll Profile. Journal of Materials Processing Technology. 125-126: 645.

[6] Zhang Jie(1990) Research on CVC roll curve and flatness. Mechanical and Engineering School of Beijing Technology University.(in Chinese)

[7] Wang Renzhong, He Anrui, Yang Quan et al(2006) Profile Control Capability of LVC Work Roll Contour. Iron and Steel. 41(5): 41-44. (in Chinese)

[8] He Wei, Di Hongshuang, Xia Xiaoming et al(2006) Design of a Five-order CVC Roll Profile. Steel Rolling. 23(2): 12-15. (in Chinese)

[9] Guo Zhongfeng, Xu Jianzhong, Li Changsheng et al(2002) Comparative Analysis of Some Typical Roll Shapes with Roll Gap Variable Through Roll Shifting. Journal of Northeastern University(Natural Science), 2008. 29(6): 830-833. (in Chinese)

[10] Zheng Lei(2009) Optimizing Design and application of roll curve in An Steel 2150mm HSM. Northeastern University. 61-67. (in Chinese) 